\title{
Neuroimaging Findings in Patients with COVID-19: A Systematic Review and Meta-Analysis
}

\author{
Pyeong Hwa Kim ${ }^{1}$, Minjae Kim², Chong Hyun Suh ${ }^{1}$, Sae Rom Chung ${ }^{1}$, Ji Eun Park ${ }^{1}$, \\ Soo Chin Kim ${ }^{1}$, Young Jun Choi ${ }^{1}$, Jeong Hyun Lee ${ }^{1}$, Ho Sung Kim ${ }^{1}$, Jung Hwan Baek ${ }^{1}$, \\ Choong Gon Choi ${ }^{1}$, Sang Joon Kim ${ }^{1}$ \\ ${ }^{1}$ Department of Radiology and Research Institute of Radiology, University of Ulsan College of Medicine, Asan Medical Center, Seoul, Korea;
}

Objective: Central nervous system involvement in coronavirus disease 2019 (COVID-19) has been increasingly reported. We performed a systematic review and meta-analysis to evaluate the incidence of radiologically demonstrated neurologic complications and detailed neuroimaging findings associated with COVID-19.

Materials and Methods: A systematic literature search of MEDLINE/PubMed and EMBASE databases was performed up to September 17, 2020, and studies evaluating neuroimaging findings of COVID-19 using brain CT or MRI were included. Several cohort-based outcomes, including the proportion of patients with abnormal neuroimaging findings related to COVID-19 were evaluated. The proportion of patients showing specific neuroimaging findings was also assessed. Subgroup analyses were also conducted focusing on critically ill COVID-19 patients and results from studies that used MRI as the only imaging modality. Results: A total of 1394 COVID-19 patients who underwent neuroimaging from 17 studies were included; among them, 3.4\% of the patients demonstrated COVID-19-related neuroimaging findings. Olfactory bulb abnormalities were the most commonly observed $(23.1 \%)$. The predominant cerebral neuroimaging finding was white matter abnormality $(17.6 \%)$, followed by acute/subacute ischemic infarction (16.0\%), and encephalopathy (13.0\%). Significantly more critically ill patients had COVID-19-related neuroimaging findings than other patients $(9.1 \%$ vs. $1.6 \% ; p=0.029)$. The type of imaging modality used did not significantly affect the proportion of COVID-19-related neuroimaging findings.

Conclusion: Abnormal neuroimaging findings were occasionally observed in COVID-19 patients. Olfactory bulb abnormalities were the most commonly observed finding. Critically ill patients showed abnormal neuroimaging findings more frequently than the other patient groups. White matter abnormalities, ischemic infarctions, and encephalopathies were the common cerebral neuroimaging findings.

Keywords: COVID-19; Neuroimaging; Computed tomography; Magnetic resonance imaging; Meta-analysis

\section{INTRODUCTION}

Following the widespread global outbreak of the novel severe acute respiratory syndrome coronavirus 2 (SARS-

Received: February 15, 2021 Revised: March 31, 2021

Accepted: April 21, 2021

Corresponding author: Chong Hyun Suh, MD, PhD, Department of Radiology and Research Institute of Radiology, University of Ulsan College of Medicine, Asan Medical Center, 88 Olympic-ro 43-gil, Songpa-gu, Seoul 05505, Korea.

- E-mail: chonghyunsuh@amc.seoul.kr This is an Open Access article distributed under the terms of the Creative Commons Attribution Non-Commercial License (https://creativecommons.org/licenses/by-nc/4.0) which permits unrestricted non-commercial use, distribution, and reproduction in any medium, provided the original work is properly cited.
CoV-2) in December 2019 [1], the World Health Organization designated coronavirus disease 2019 (COVID-19) as a global pandemic. COVID-19 has been shown to have multiorgan manifestations. Specifically, central nervous system (CNS) involvement has been increasingly reported. Although the exact pathophysiology remains controversial $[2,3]$, neurologic complications can have a striking impact on patient management. Indeed, when ischemic or hemorrhagic stroke develops, patients should undergo dedicated treatment (similar to patients without COVID-19) as well as require treatment for infection control $[4,5]$. Management of encephalopathy associated with COVID-19 is also challenging, as patients with encephalopathy have a longer hospitalization period, worse functional impairment, 
and higher 30-day mortality rate [6].

Therefore, it is important for clinicians to identify the number of patients with neuroradiological manifestations during hospitalization and understand how these may appear. Many case studies have recently been published [7-12], which can be analyzed to better understand CNS involvement in COVID-19. Several relevant meta-analyses have recently been published, but most of the studies merely focused on clinical manifestations of COVID-19 (e.g., headache, smell disturbances, dizziness) [13-17]. One metaanalysis focused on neuroimaging findings of COVID-19 [18], but only four types of neuroimaging findings were evaluated (i.e., cerebral infarction, cerebral microhemorrhages, intracranial hemorrhage, and encephalitis/encephalopathy). In addition, the cohort-based outcomes of all COVID-19 patients, not just the neuroimaging cohort, were evaluated.

Herein, we performed a systematic review and metaanalysis to evaluate the incidence of radiologically proven neurologic complications and detail the neuroimaging findings associated with COVID-19.

\section{MATERIALS AND METHODS}

This study was conducted according to the guidelines of the preferred reporting items for systematic reviews and meta-analyses (PRISMA) [19].

\section{Search Strategy and Eligibility Criteria}

A literature search of the MEDLINE/PubMed and EMBASE databases was conducted using pertinent MeSH or Emtree terms with common keywords for relevant articles until September 17, 2020. The search terms were as follows: ((COVID-19) OR (coronavirus) OR (SARS-CoV-2) OR (2019nCoV)) AND ((brain) OR (neuro) OR (neuroimaging) OR (neurologic*) OR (nervous system) OR (olfactory)) AND ((magnetic resonance imaging) OR (MR imaging) OR (MRI)). The search was not limited by language, human or animal studies, or publication dates.

After eliminating duplicates, the articles were screened based on their titles and abstracts. The full article texts were then assessed according to the following eligibility criteria: 1) population: patients with COVID-19;2) index test: brain CT or MRI conducted during hospitalization or admission to the emergency department due to COVID-19; 3) comparator(s)/control: not applicable; 4) outcomes: neuroimaging findings associated with COVID-19; and 5) study design: not limited. We excluded studies that met any of the following criteria: 1 ) review; 2) case reports or case series including fewer than 10 patients; 3 ) guidelines; 4) letters without original data, editorials, reply, corrections, and comments; 5) animal studies; 6) study protocol; and 7) studies with partially overlapping patient cohorts (for studies with overlapping study populations, the study with the largest population was selected). The literature search and criteria application were conducted independently by two authors (with 3 and 7 years of experience in performing systematic reviews and meta-analyses, respectively). Any discrepancies were resolved by consensus.

\section{Data Extraction and Quality Assessment}

A standardized extraction form prepared in Microsoft Excel was used to obtain the following information from the selected studies: 1) study characteristics: study location, institution, study period, and study design (prospective vs. retrospective; multicenter vs. single-center; consecutive enrollment); 2) patient characteristics: sample size, inclusion criteria for each study, number of patients who underwent neuroimaging, types of neuroimaging studies (CT and/or MRI), and proportion of critically ill patients;

3) cohort-based outcomes: number of all COVID-19 patients in each hospital cohort, number of patients with neurologic symptoms, number of patients with neuroimaging data, number of patients with any abnormal neuroimaging findings, and number of patients with neuroimaging findings related to COVID-19; and 4) detailed neuroimaging findings. Neuroimaging findings were considered not related to COVID-19 if they were likely chronic lesions, such as cavernoma, chronic infarcts, known white matter lesions from multiple sclerosis, white matter lesions of small vessel disease, and microbleeds associated with chronic infarction. The spectrum of abnormal neuroimaging findings was classified into several categories, including white matter abnormalities, gray matter abnormalities, encephalopathy (regardless of white/gray matter involvement), cerebral microbleeds, intracranial hemorrhage, olfactory bulb abnormality, cranial neuropathy other than olfactory nerves, and others. The quality of evidence in the included studies was independently assessed by two authors using the US National Institutes of Health Quality Assessment of Case Series Studies tool [20]. Any discrepancies were resolved by consensus.

\section{Data Synthesis and Analysis}

The cohort-based outcomes were as follows: 1) 
proportion of patients with neurologic symptoms among all COVID-19 patients; 2) proportion of patients who underwent neuroimaging examinations among patients with neurological symptoms; 3 ) proportion of patients underwent neuroimaging examinations among all patients; 4) proportion of patients with neuroimaging findings among patients underwent neuroimaging examinations; 5) proportion of patients with neuroimaging findings related to COVID-19 among patients underwent neuroimaging examinations; and 6) proportion of patients with neuroimaging findings related to COVID-19 among all patients (Fig. 1). The proportion of patients showing specific neuroimaging findings among patients who underwent neuroimaging examinations was also an outcome of interest. Meta-analytic pooling was performed based on the inverse variance method for calculating weights, and pooled estimates with their $95 \%$ confidence intervals (CIs) were determined using DerSimonian-Laird randomeffects modeling. Heterogeneity across studies was assessed using $Q$ tests and $\mathrm{I}^{2}$ statistics, with $\mathrm{I}^{2}>50 \%$ indicating the presence of heterogeneity [21-23]. Publication bias was evaluated using funnel plots and Egger's test when the number of analyzed studies was 10 or more [24,25].

We conducted a subgroup analysis of critically ill COVID-19 patients. Patients were considered critically ill

COVID-19 (C)

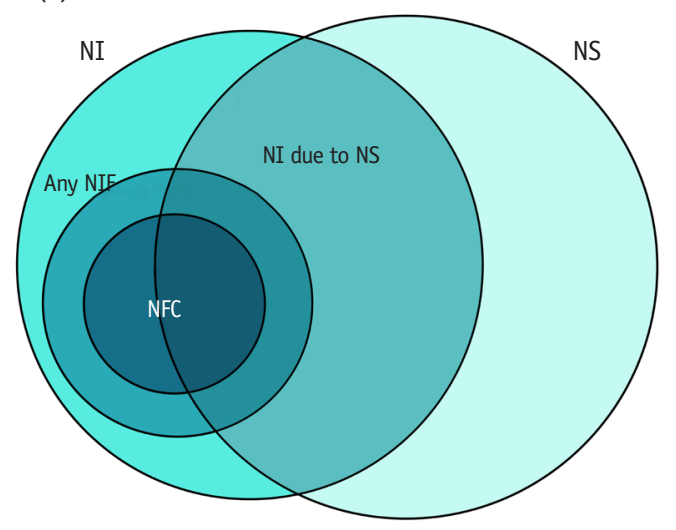

Fig. 1. Venn diagram illustrating the cohort-based outcomes used in this study. 1) proportion of patients with NS among all COVID-19 patients (NS/C); 2) proportion of patients with NI among patients with NI-NS; 3 ) proportion of patients who underwent NI among all patients assessed (NI/C); 4) proportion of patients with any NI findings among the patients who underwent NI (NF/NI); 5) proportion of patients with NFC among the patients who underwent NI (NFC/NI); 6) proportion of patients with NFC among all patients assessed (NFC/C). COVID-19 = coronavirus disease 2019, NF = NI findings, NFC $=\mathrm{NF}$ related to COVID-19, NI = neuroimaging, NS = neurologic symptoms if they met any of the following criteria: 1) respiratory failure requiring mechanical ventilation, 2) shock, or 3) combined failure of other organs requiring intensive care unit (ICU) monitoring [26]. A meta-regression analysis was performed to determine whether COVID-19 severity was a source of heterogeneity. In addition, a subgroup analysis including studies using only MRI as an imaging modality was performed. Two-sided tests were used, and statistical significance was set at $p<0.05$. All statistical analyses were performed by one of the authors using $R$ software (version 3.1.2; R Foundation for Statistical Computing) with the "meta" and "metafor" packages.

\section{RESULTS}

\section{Literature Search}

A flowchart of the publication selection process is presented in Figure 2. A total of 216 non-duplicate studies were identified. Of these, 188 articles were excluded after title and abstract review, and 11 studies were further excluded for the following reasons: 1$)$ studies with partially overlapping patient cohorts $(n=4) ; 2)$ studies without neuroimaging findings $(n=3) ; 3$ ) population-based study $(\mathrm{n}=1) ; 4)$ study not yet accepted by a peer-reviewed journal $(n=1) ; 5)$ study on recovered COVID-19 patients $(n=1)$; and 6) postmortem study $(n=1)$. Consequently, the analysis included a total of 17 studies comprising 1394 COVID-19 patients who underwent brain CT/MRI during hospitalization or admission in the emergency department due to COVID-19 met the eligibility criteria and were included in the analysis.

\section{Characteristics of the Included Studies}

The detailed study and patient characteristics are summarized in Table 1. All studies were conducted between February and June 2020 in the US $[9,11,12,27-31]$ and Europe $[7,8,10,32-37]$. Except for the study by Eliezer et al. [34], all studies were retrospective. Three included studies focused on specific symptoms of the diseases, including olfactory function loss [34], acute ischemic stroke [29], and cerebrovascular disease (e.g., cerebral ischemia, intracerebral hemorrhage, and posterior reversible encephalopathy syndrome-like leukoencephalopathy) [35]. Seven studies analyzed only brain MRI findings $[8,27,28,30,33,34,36]$, while the other 10 also analyzed CT findings $[7,9-12,29,31,32,35,37]$. Data on COVID-19 severity were available in eight studies [9,29-33,36,37]; 


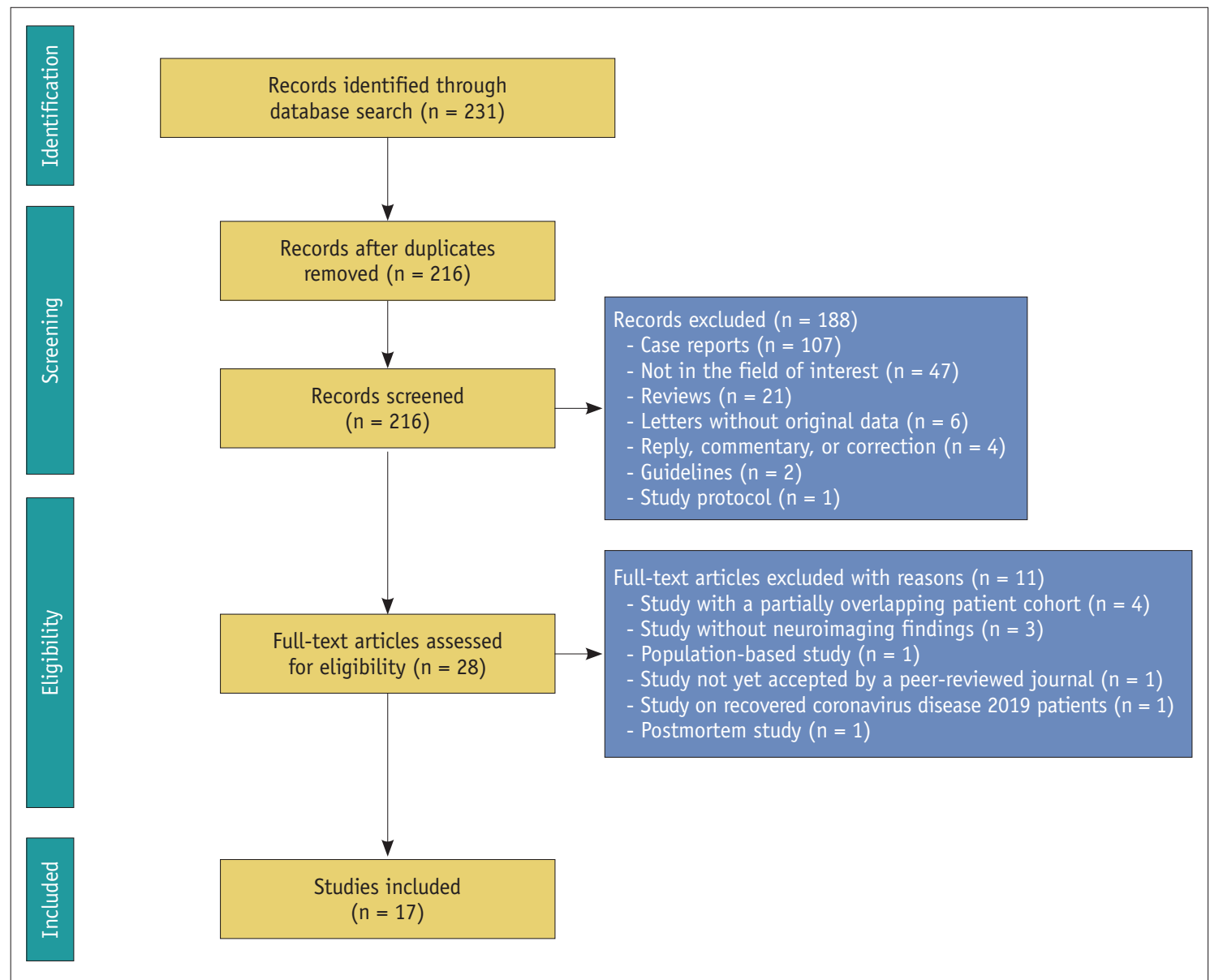

Fig. 2. Flow chart depicting the study selection process.

of those, four included only critically ill COVID-19 patients who required ICU care [30-32,36]. The quality of these studies was good $(n=12)[7-9,11,12,28,29,32-35,37]$ or fair $(n=5)[10,27,30,31,36]$ (Supplementary Table 1).

\section{Neuroimaging Cohort}

Four of the 17 included studies [31-33,36] (one study on all COVID-19 patients [33] and three studies on critically ill COVID-19 patients $[31,32,36]$ ) reported the proportion of patients with neurologic symptoms among all COVID-19 patients; the proportion of patients with neurologic symptoms ranged from $21.3-55.6 \%$, with a meta-analytic pooled proportion of $32.4 \%$ (95\% CI, 22.9-43.7\%). Of those, neuroimaging studies were performed in $23.7-100 \%$ of patients (pooled proportion, 72.4\% [95\% CI, 36.0$92.5 \%]$ ), showing a high discrepancy across the studies.

Regardless of the presence of acute neurologic symptoms requiring neuroimaging, neuroimaging studies were performed in $2.1-55.6 \%$ of COVID-19 patients from each study population $[7,9,11,12,27,28,31-33,36]$, with a meta- analytic pooled proportion of $9.7 \%$ (95\% CI, $5.8-15.9 \%$ ). No significant publication bias was observed $(p=0.820)$.

Among patients who underwent neuroimaging studies, 58.9-86.4\% showed abnormal neuroimaging findings [27,28,31-33], with a meta-analytic pooled proportion of $73.6 \%$ (95\% CI, 62.2-82.6\%). After excluding neuroimaging findings possibly not related to COVID-19 or associated comorbidities, the pooled proportion decreased to $35.5 \%$ (95\% CI, 24.4-48.3\%). Consequently, 3.4\% (95\% CI, 1.4-8.2\%) of all COVID-19 patients demonstrated abnormal neuroimaging findings related to COVID-19.

\section{Abnormal Neuroimaging Findings}

The abnormal neuroimaging findings of the included neuroimaging cohorts are summarized in Table 2 and Figure 3. Two studies reported the proportion of patients with olfactory bulb abnormalities $[7,9]$, with these abnormalities showing the highest meta-analytic pooled proportion (23.1\%; 95\% CI, $12.9-37.7 \%)$. The most common cerebral neuroimaging finding was white matter abnormalities 


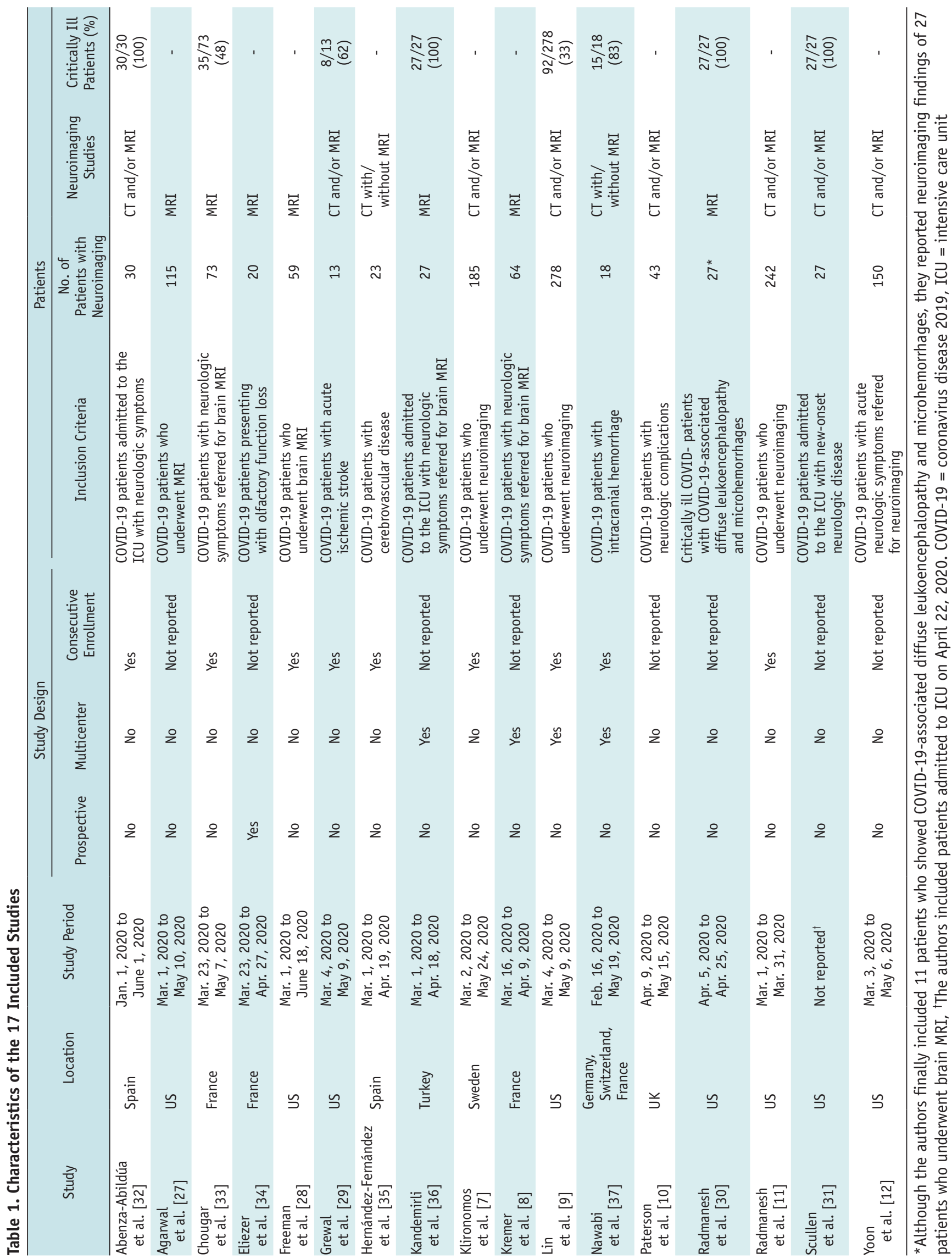


Table 2. Summary of Neuroimaging Findings in Patients with COVID-19

\begin{tabular}{|c|c|c|c|c|}
\hline Neuroimaging Findings & Detailed Findings Included & $\begin{array}{l}\text { No. } \\
\text { of Studies }\end{array}$ & $\begin{array}{c}\text { No. } \\
\text { of Patients (\%) }\end{array}$ & $\begin{array}{c}\text { Meta-Analytic } \\
\text { Proportion }(95 \% \mathrm{CI})\end{array}$ \\
\hline Olfactory bulb abnormality & T2 hyperintensity in the olfactory bulbs/tracts & 2 & $11 / 49(22)$ & $23.1(12.9-37.7)$ \\
\hline White matter abnormality & $\begin{array}{l}\text { White matter changes, leukoencephalopathy, } \\
\text { multifocal enhancing white matter lesions, } \\
\text { COVID-19-related disseminated } \\
\text { leukoencephalopathy }\end{array}$ & 6 & $79 / 465(17)$ & $17.6(7.5-36.0)^{*}$ \\
\hline $\begin{array}{l}\text { Acute or subacute } \\
\text { ischemic infarction }\end{array}$ & - & 10 & $130 / 989(13)$ & $16.0(10.3-23.9)^{*}$ \\
\hline $\begin{array}{l}\text { Encephalopathy (regardless } \\
\text { of white/grey matter } \\
\text { involvement) }\end{array}$ & $\begin{array}{l}\text { COVID-19-associated encephalopathy, } \\
\text { cytotoxic lesions of the corpus callosum, } \\
\text { limbic encephalitis, acute hemorrhagic } \\
\text { necrotizing encephalopathy, miscellaneous } \\
\text { encephalitis }\end{array}$ & 8 & $58 / 432(13)$ & $13.0(4.7-31.3)^{*}$ \\
\hline Cerebral microbleeds & - & 8 & $107 / 983(11)$ & $12.1(5.0-26.3)^{*}$ \\
\hline Intracranial hemorrhage & $\begin{array}{l}\text { Intraparenchymal hemorrhage, subdural } \\
\text { hemorrhage, subarachnoid hemorrhage }\end{array}$ & 6 & 47/766 (6) & $7.8(3.7-16.0)^{*}$ \\
\hline Others & $\begin{array}{l}\text { Venous thrombosis, PRES, ADEM, } \\
\text { seizure-related perfusion abnormalities, } \\
\text { isolated perfusion abnormalities, metabolic } \\
\text { abnormalities, leptomeningeal enhancement }\end{array}$ & 5 & $38 / 472(8)$ & $7.4(1.9-24.7)^{*}$ \\
\hline Venous thrombosis & - & 2 & $3 / 57(5)$ & $5.5(1.8-15.6)$ \\
\hline PRES & - & 2 & $5 / 351(1)$ & $1.6(0.6-3.8)$ \\
\hline Grey matter abnormality & $\begin{array}{l}\text { Abnormal basal ganglia signal, cortical T2 } \\
\text { hyperintensity }\end{array}$ & 2 & $11 / 86(13)$ & $10.2(0.4-78.2)$ \\
\hline $\begin{array}{l}\text { Cranial neuropathy other than } \\
\text { olfactory nerves }\end{array}$ & $\begin{array}{l}\text { T2 hyperintensity and/or enhancement in the } \\
\text { optic (II), oculomotor (III), facial (VII), and } \\
\text { vestibulocochlear nerves (VIII) }\end{array}$ & 3 & $6 / 371(2)$ & $2.7(0.6-11.5)^{*}$ \\
\hline
\end{tabular}

We excluded neuroimaging findings not likely to be related to COVID-19, including cavernoma, chronic infarcts, known white matter lesions from multiple sclerosis, white matter lesions of small vessel disease, and microbleeds associated with chronic infarction. Neuroimaging findings were ordered from the top in the order of decreasing meta-analytic proportions. *The pooled proportion showed substantial heterogeneity $\left(\mathrm{I}^{2}>50 \%\right)$. ADEM = acute disseminated encephalomyelitis, $\mathrm{CI}=$ confidence interval, $\mathrm{COVID}-19=$ coronavirus disease 2019, PRES = posterior reversible encephalopathy syndrome

(17.6\%; 95\% CI, 7.5-36.0\%), followed by acute/subacute ischemic infarction $(16.0 \% ; 95 \%$ CI, 10.3-23.9\%), encephalopathy $(13.0 \% ; 95 \% \mathrm{CI}, 4.7-31.3 \%)$, cerebral microbleeds $(12.1 \% ; 95 \%$ CI, $5.0-26.3 \%)$, and intracranial hemorrhage $(7.8 \% ; 95 \% \mathrm{CI}, 3.7-16.0 \%)$. There was no significant publication bias in the pooled estimates of acute/subacute ischemic infarction $(p=0.725)$.

Acute or subacute ischemic infarction was present in 168 patients in 13 studies [7-12,28-31,33,35,36]. Of those, data regarding Trial of ORG 10172 in Acute Stroke Treatment (TOAST) classification were available for 59 patients from three studies $[9,29,35]$ The most common classification was cryptogenic $(54 \% ; 32 / 59)$, followed by cardioembolism $(34 \% ; 20 / 59)$, large artery atherosclerosis $(5 \% ; 3 / 59)$, other determined etiology $(5 \% ; 3 / 59)$, and small-vessel occlusion $(2 \% ; 1 / 59)$. Of the 79 patients with available infarction data, 35 (44\%) had large vessel occlusion. Data on infarction territory were available for 24 patients from four studies $[10,12,31,36]$; the most common territory was the middle cerebral artery $(46 \% ; 11 / 24)$, followed by multiple territories $(21 \% ; 5 / 24)$, the posterior cerebral artery $(17 \% ; 4 / 24)$, the posterior-inferior cerebellar artery $(8 \% ; 2 / 24)$, and the watershed zone $(8 \% ; 2 / 24)$. Among the 30 patients for whom prognosis data were available, 14 (47\%) showed favorable outcomes.

White matter abnormalities or encephalopathy were present in 120 patients from 11 studies $[7,8,10,12,27,28,30-33,35]$. Several characteristic forms were reported in 29 patients, including diffuse/ disseminated leukoencephalopathy $(n=16)$, cytotoxic 


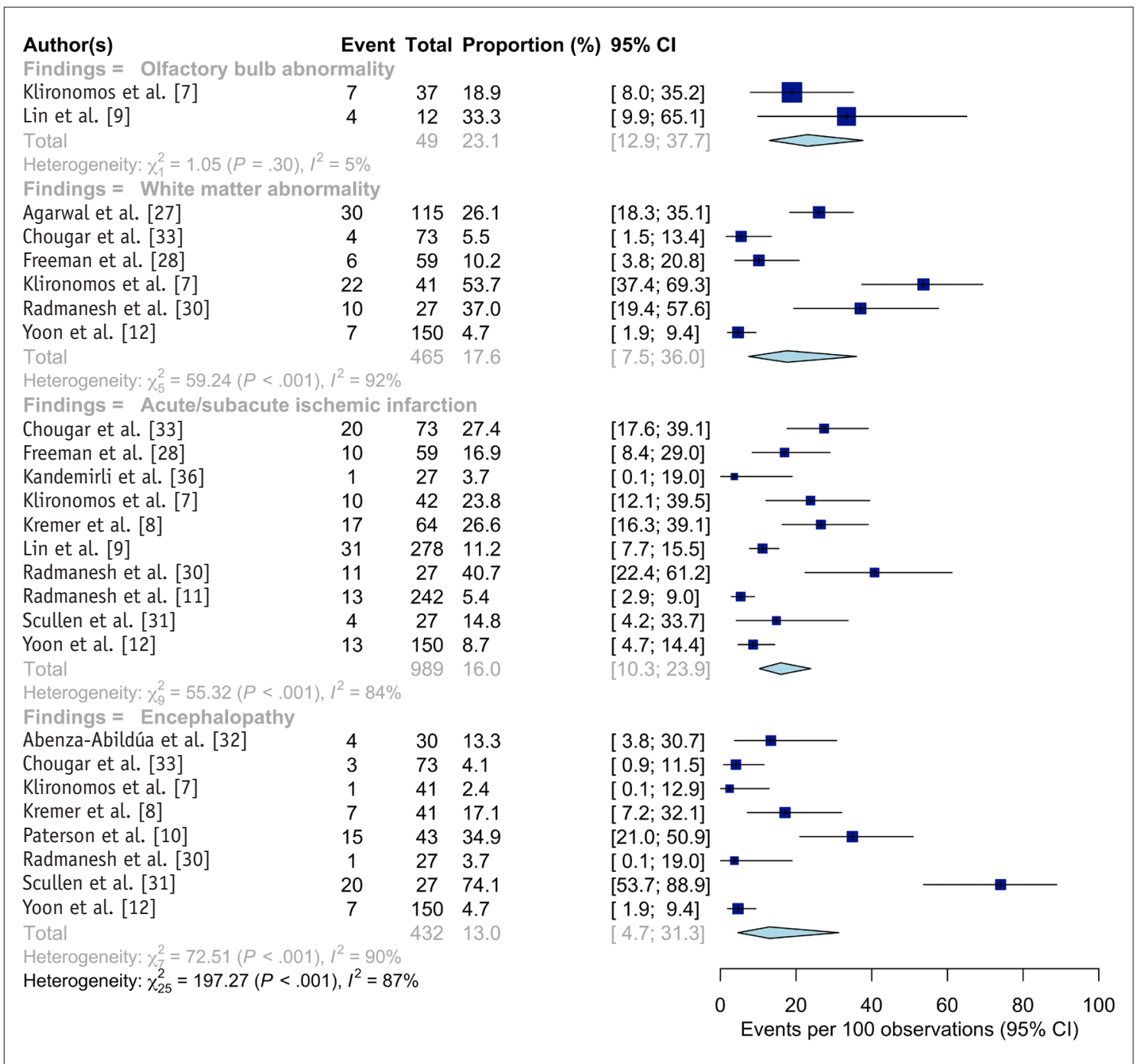

Fig. 3. Forest plots representing the proportion of the patients with specific neuroimaging findings among the patients who underwent neuroimaging studies. Pooled estimates with $95 \%$ CIs were determined using DerSimonian-Laird random-effects modeling. CI = confidence interval

lesion of the corpus callosum $(n=5)$, acute hemorrhagic necrotizing encephalopathy $(n=4)$, and limbic encephalitis $(n=4)$. Prognosis was only available in the study by

Paterson et al. [10]. One patient with acute hemorrhagic necrotizing encephalopathy initially underwent intravenous methylprednisolone, but her consciousness level decreased, and she subsequently underwent emergent craniectomy. She then received oral prednisolone $60 \mathrm{mg}$ daily and 5 days of intravenous immunoglobulin (IVIG), and finally showed clinical improvement. Two patients with limbic encephalitis received IVIG and showed incomplete improvement.

Intracranial hemorrhage was present in 65 patients from seven studies $[7,9,11,12,30,32,37]$. Of those, data regarding the types of hemorrhage were available for 54 patients from six studies $[7,9,12,30,32,37]$. The most common type was intracerebral hemorrhage (44\%; 24/54), followed by subarachnoid hemorrhage $(35 \% ; 19 / 54)$ and subdural hemorrhage $(24 \%$; $13 / 54)$ (some patients had multiple types of hemorrhage), with 13 patients showing an intraventricular extension of the hemorrhage.

Radiologic olfactory bulb abnormalities were present in 11 patients in two studies [7,9]. Lin et al. [9] reported an increased signal on postcontrast T2 fluid-attenuated inversion recovery (FLAIR) imaging in four of 12 patients, one of whom had documented anosmia. No abnormalities were reported along the olfactory cleft. Klironomos et al. [7] reported an increased signal on postcontrast T2 FLAIR images in seven of 37 patients (19\%). The presence or absence of anosmia in these patients was not reported. Eliezer et al. [34] reported 20 COVID-19 patients with 
olfactory function loss, of whom 19 (95\%) showed complete obstruction of the olfactory clefts on MRI. These findings were persistent at the 1-month follow-up in seven patients $(35 \%)$, with a correlation between olfactory score and olfactory cleft obstruction. However, none of the patients showed olfactory bulb abnormalities.

\section{Subgroup Analysis: Critically Ill Patients}

The cohort-based results of subgroup analysis of critically ill patients, including four studies and 111 critically ill COVID-19 patients [30-32,36], are summarized in Supplementary Table 2.

Compared to the other studies, the proportion of patients who underwent neuroimaging studies was significantly higher in studies on critically ill patients $(22.7 \%$ vs. $6.8 \% ; p=$ $0.027)$. In addition, the proportion of patients with abnormal neuroimaging findings related to COVID-19 among all patients evaluated in this study was significantly higher in critically ill patients $(9.1 \%$ vs. $1.6 \% ; p=0.029)$. Other cohort-based outcomes did not show any significant differences.

The abnormal neuroimaging findings of critically ill patients are also summarized in Supplementary Table 2. Encephalopathy (22.2\%) and acute or subacute ischemic infarction (17.2\%) were the most common cerebral neuroimaging findings. The proportion of each neuroimaging finding in critically ill patients, including those with ischemic infarction, did not differ significantly.

\section{Subgroup Analysis: MRI}

The cohort-based results of subgroup analysis, including studies using only MRI as an imaging modality, are summarized in Supplementary Table 3.

The proportion of patients with abnormal neuroimaging findings related to COVID-19 among patients who underwent neuroimaging studies did not differ, whether only MRI was used or not $(40.1 \%$ vs. $31.1 \% ; p=0.397)$. In contrast, the proportion of patients who underwent neuroimaging among the patients with neurological symptoms $(37.1 \%$ vs. $98.3 \% ; p<0.001)$, the proportion of patients who underwent neuroimaging among all COVID-19 patients $(3.4 \%$ vs. $18.5 \% ; p<0.001)$, and the proportion of patients with abnormal neuroimaging findings related to COVID-19 when the denominator was set to be all COVID-19 patients $(0.9 \%$ vs. $8.4 \% ; p=0.002)$ was significantly lower when only MRI was used as an imaging modality. Regarding detailed neuroimaging findings, acute or subacute ischemic infarction was more frequently observed when only MRI was used as an imaging modality $(24.4 \%$ vs. $11.0 \% ; p=0.011)$.

\section{DISCUSSION}

This meta-analysis of 17 studies reports that $9.7 \%$ of COVID-19 patients underwent neuroimaging studies and that $35.5 \%$ of them had neuroimaging findings associated with COVID-19. Consequently, 3.4\% of COVID-19 patients undergoing neuroimaging demonstrated neuroimaging findings related to COVID-19. Olfactory bulb abnormality was the most common neuroimaging finding (23.1\%). The predominant cerebral neuroimaging finding was white matter abnormality $(17.6 \%)$, followed by acute/subacute ischemic infarction (16.0\%), and encephalopathy (13.0\%). Intracranial hemorrhage and cerebral microbleeds were observed in $7.8 \%$ and $12.1 \%$ of the patients, respectively. Critically ill patients underwent neuroimaging studies more frequently $(22.7 \%$ vs. $6.8 \% ; p=0.027)$ and presented more frequent COVID-19-related neuroimaging findings when compared in all COVID-19 patients evaluated in this study ( $9.1 \%$ vs. $1.6 \% ; p=0.029)$. Although MRI was less frequently performed in COVID-19 patients (3.4\% vs. $18.5 \% ; p<0.001)$, the proportion of patients with COVID19 -related abnormal neuroimaging findings was similar regardless of the imaging modality used.

Our results showed that COVID-19-related neuroimaging findings were more frequently observed in critically ill patients than the other patient groups. This finding may emphasize the role of neuroimaging in critically ill patients. Since neurologic deficits may be masked in critically ill patients due to decreased levels of consciousness, it may be necessary to actively perform neuroimaging studies in critically ill patients in whom neurologic complications are suspected.

In this study, there were no significant differences between the proportions of patients with ischemic infarction in studies that included only critically ill patients and other studies ( $17.2 \%$ vs. $15.1 \% ; p=0.617)$. This finding indicates that ischemic infarction may not occur due to the systemic inflammatory process accompanying acute respiratory distress syndrome (ARDS) and that it may be a consequence of the hypercoagulable state and thrombosis. Indeed, $44 \%$ of the patients with ischemic infarction in this study had large vessel occlusion, a proportion higher than that reported in the general population (24-46\%) [38-41]. This observation has also been suggested in the existing literature; Kremer et al. [8] reported lower oxygen 
demand and ARDS in patients with ischemic infarction, while Beyrouti et al. [42] reported that all six patients with ischemic infarction in their study were in prothrombotic states and had large vessel occlusion. However, this evidence is insufficient to elucidate a causal relationship between ischemic infarction and hypercoagulability, and further investigation is required.

Another important neurological manifestation of COVID-19 is anosmia. Although the current meta-analysis included only two studies on anosmia, olfactory bulb abnormalities were relatively common, occurring in approximately $23.1 \%$ of patients who underwent neuroimaging. However, it remains controversial whether anosmia in COVID-19 occurs due to olfactory cleft congestion or direct olfactory nerve damage. SARS-CoV-2 has been shown to invade target cells through interactions between the angiotensin-converting enzyme 2 (ACE2) protein and the S protein of the target cell $[43,44]$. SARS-CoV-2 may enter through the sustentacular cells of the olfactory epithelium (which express ACE2 receptors) [44], consequently causing structural changes in the olfactory bulb. However, among the 20 COVID-19 patients with anosmia investigated, Eliezer et al. [34] reported olfactory cleft obstruction in $95 \%$ of the cases but without any olfactory bulb abnormalities. Thus, it could be hypothesized that olfactory cleft obstruction occurs first, followed by the secondary involvement of the central olfactory pathway. However, further investigation is required to clarify this issue.

This study has several limitations. First, all studies, except one, were retrospective in their design, which confers a risk of selection bias. Second, the sample size was moderate and resulted in underpowered statistical analyses. Third, considerable between-study variability was observed in most of the study outcomes, which could have resulted from differences in cohort characteristics, regional spread of COVID-19, institutional policies, institutional neuroradiologists' perspectives, and neuroimaging study types and protocols. In particular, the proportion of patients among the COVID-19 patients who underwent neuroimaging was highly variable across the studies (23.7$100 \%$ ). Although we performed a subgroup analysis of the studies that only used MRI, the substantial heterogeneity of our cohort could not be completely resolved. Fourth, the terms describing a specific neuroimaging finding (e.g., white matter change, leukoencephalopathy, COVID-19related diffuse leukoencephalopathy) were heterogeneous across studies, complicating the meta-analytic pooling.
Fifth, it is unclear how the authors concluded whether microbleeds were caused by COVID-19 or small vessel disease. Lastly, although we conducted the analysis after excluding chronic neuroimaging findings, it is difficult to guarantee that acute or subacute neuroimaging findings are directly associated with COVID-19. Nevertheless, our results may provide useful information for understanding the neuroimaging findings in COVID-19.

In conclusion, active neuroimaging studies are recommended in critically ill patients, considering the high proportion of patients presenting with neuroimaging findings related to COVID-19. The predominant cerebral neuroimaging finding was white matter abnormalities, followed by acute/subacute ischemic infarctions, and encephalopathies. Olfactory bulb abnormalities are also frequently observed in COVID-19 patients.

\section{Supplement}

The Supplement is available with this article at https://doi.org/10.3348/kjr.2021.0127.

\section{Conflicts of Interest}

The authors have no potential conflicts of interest to disclose.

\section{Author Contributions}

Conceptualization: Chong Hyun Suh. Data curation: Pyeong Hwa Kim, Minjae Kim, Chong Hyun Suh. Formal analysis: Pyeong Hwa Kim, Chong Hyun Suh. Investigation: Pyeong Hwa Kim, Chong Hyun Suh. Methodology: Pyeong Hwa Kim, Chong Hyun Suh. Project administration: Pyeong Hwa Kim, Chong Hyun Suh. Resources: all authors. Software: Pyeong Hwa Kim, Chong Hyun Suh. Supervision: Chong Hyun Suh, Sang Joon Kim. Validation: Chong Hyun Suh. Visualization: Pyeong Hwa Kim. Writing_original draft: Pyeong Hwa Kim, Minjae Kim. Writing—review \& editing: all authors.

\section{ORCID iDs}

Pyeong Hwa Kim

https://orcid.org/0000-0003-4276-8803

Minjae Kim

https://orcid.org/0000-0002-5382-9360

Chong Hyun Suh

https://orcid.org/0000-0002-4737-0530 


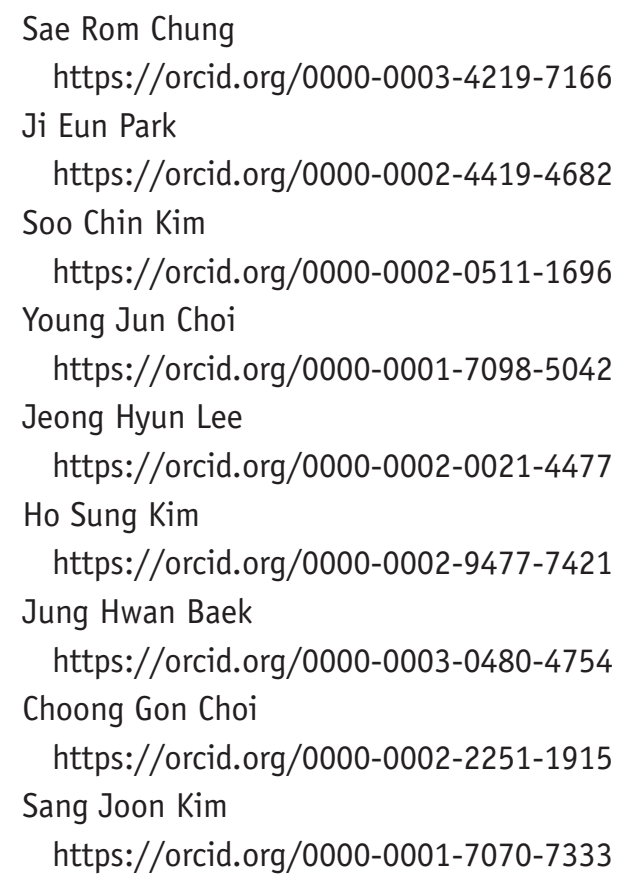

\section{REFERENCES}

1. Zhou P, Yang XL, Wang XG, Hu B, Zhang L, Zhang W, et al. A pneumonia outbreak associated with a new coronavirus of probable bat origin. Nature 2020;579:270-273

2. Zubair AS, McAlpine LS, Gardin T, Farhadian S, Kuruvilla DE, Spudich S. Neuropathogenesis and neurologic manifestations of the coronaviruses in the age of coronavirus disease 2019: a review. JAMA Neurol 2020;77:1018-1027

3. Mao L, Jin H, Wang M, Hu Y, Chen S, He Q, et al. Neurologic manifestations of hospitalized patients with coronavirus disease 2019 in Wuhan, China. JAMA Neurol 2020;77:683-690

4. Wira CR, Goyal M, Southerland AM, Sheth KN, McNair ND, Khosravani $\mathrm{H}$, et al. Pandemic guidance for stroke centers aiding COVID-19 treatment teams. Stroke 2020;51:2587-2592

5. Leira EC, Russman AN, Biller J, Brown DL, Bushnell CD, Caso V, et al. Preserving stroke care during the COVID-19 pandemic: potential issues and solutions. Neurology 2020;95:124-133

6. Liotta EM, Batra A, Clark JR, Shlobin NA, Hoffman SC, Orban ZS, et al. Frequent neurologic manifestations and encephalopathy-associated morbidity in Covid-19 patients. Ann Clin Transl Neurol 2020;7:2221-2230

7. Klironomos S, Tzortzakakis A, Kits A, Öhberg C, Kollia E, Ahoromazdae $A$, et al. Nervous system involvement in coronavirus disease 2019: results from a retrospective consecutive neuroimaging cohort. Radiology 2020;297:E324-E334

8. Kremer S, Lersy F, Anheim M, Merdji H, Schenck M, Oesterlé $\mathrm{H}$, et al. Neurologic and neuroimaging findings in patients with COVID-19: a retrospective multicenter study. Neurology 2020;95:e1868-e1882

9. Lin E, Lantos JE, Strauss SB, Phillips CD, Campion TR Jr, Navi
BB, et al. Brain imaging of patients with COVID-19: findings at an academic institution during the height of the outbreak in New York City. AJNR Am J Neuroradiol 2020;41:2001-2008

10. Paterson RW, Brown RL, Benjamin L, Nortley R, Wiethoff $S$, Bharucha $T$, et al. The emerging spectrum of COVID-19 neurology: clinical, radiological and laboratory findings. Brain 2020;143:3104-3120

11. Radmanesh A, Raz E, Zan E, Derman A, Kaminetzky M. Brain imaging use and findings in COVID-19: a single academic center experience in the epicenter of disease in the United States. AJNR Am J Neuroradiol 2020;41:1179-1183

12. Yoon BC, Buch K, Lang M, Applewhite BP, Li MD, Mehan WA $\mathrm{Jr}$, et al. Clinical and neuroimaging correlation in patients with COVID-19. AJNR Am J Neuroradiol 2020;41:1791-1796

13. Favas TT, Dev P, Chaurasia RN, Chakravarty K, Mishra R, Joshi D, et al. Neurological manifestations of COVID-19: a systematic review and meta-analysis of proportions. Neurol Sci 2020;41:3437-3470

14. Collantes MEV, Espiritu AI, Sy MCC, Anlacan VMM, Jamora RDG. Neurological manifestations in COVID-19 infection: a systematic review and meta-analysis. Can J Neurol Sci 2021;48:66-76

15. Nazari S, Azari Jafari A, Mirmoeeni S, Sadeghian S, Heidari $M E$, Sadeghian $S$, et al. Central nervous system manifestations in COVID-19 patients: a systematic review and meta-analysis. Brain Behav 2021;11:e02025

16. Pinzon RT, Wijaya V0, Buana RB, Al Jody A, Nunsio PN. Neurologic characteristics in coronavirus disease 2019 (COVID-19): a systematic review and meta-analysis. Front Neurol 2020;11:565

17. Chen X, Laurent S, Onur OA, Kleineberg NN, Fink GR, Schweitzer $F$, et al. A systematic review of neurological symptoms and complications of COVID-19. J Neurol 2021;268:392-402

18. Choi Y, Lee MK. Neuroimaging findings of brain MRI and CT in patients with COVID-19: a systematic review and metaanalysis. Eur J Radiol 2020;133:109393

19. Liberati A, Altman DG, Tetzlaff J, Mulrow C, Gøtzsche PC, Ioannidis JP, et al. The PRISMA statement for reporting systematic reviews and meta-analyses of studies that evaluate healthcare interventions: explanation and elaboration. $B M J$ 2009;339:b2700

20. National Institutes of Health. Quality assessment tool for case series studies. Nhlbi.nih.gov Web site. https://www.nhlbi.nih. gov/health-topics/study-quality-assessment-tools. Accessed September 24, 2020

21. Higgins JP, Thompson SG, Deeks JJ, Altman DG. Measuring inconsistency in meta-analyses. BMJ 2003;327:557-560

22. Kim KW, Lee J, Choi SH, Huh J, Park SH. Systematic review and meta-analysis of studies evaluating diagnostic test accuracy: a practical review for clinical researchers-part II. Statistical methods of meta-analysis. Korean J Radiol 2015; 16:1175-1187

23. Lee J, Kim KW, Choi SH, Huh J, Park SH. Systematic review 
and meta-analysis of studies evaluating diagnostic test accuracy: a practical review for clinical researchers-part II. Statistical methods of meta-analysis. Korean J Radiol 2015;16:1188-1196

24. Egger M, Davey Smith G, Schneider M, Minder C. Bias in meta-analysis detected by a simple, graphical test. $B M J$ 1997;315:629-634

25. Sterne JA, Egger M, Smith GD. Systematic reviews in health care: investigating and dealing with publication and other biases in meta-analysis. BMJ 2001;323:101-105

26. National Health Commission of the People's Republic of China. Novel coronavirus pneumonia diagnosis and treatment plan (provisional 7th edition). Chinalawtranslate.com Web site. https://www.chinalawtranslate.com/en/coronavirustreatment-plan-7/. Accessed March 22, 2021

27. Agarwal S, Jain R, Dogra S, Krieger P, Lewis A, Nguyen V, et al. Cerebral microbleeds and leukoencephalopathy in critically ill patients with COVID-19. Stroke 2020;51:2649-2655

28. Freeman CW, Masur J, Hassankhani A, Wolf RL, Levine JM, Mohan S. Coronavirus disease (COVID-19)-related disseminated leukoencephalopathy: a retrospective study of findings on brain MRI. AJR Am J Roentgenol 2021;216:10461047

29. Grewal P, Pinna P, Hall JP, Dafer RM, Tavarez T, Pellack DR, et al. Acute ischemic stroke and COVID-19: experience from a comprehensive stroke center in Midwest US. Front Neurol 2020;11:910

30. Radmanesh A, Derman A, Lui YW, Raz E, Loh JP, Hagiwara M, et al. COVID-19-associated diffuse leukoencephalopathy and microhemorrhages. Radiology 2020;297:E223-E227

31. Scullen T, Keen J, Mathkour M, Dumont AS, Kahn L. Coronavirus 2019 (COVID-19)-associated encephalopathies and cerebrovascular disease: the new Orleans experience. World Neurosurg 2020;141:e437-e446

32. Abenza-Abildúa MJ, Ramírez-Prieto MT, Moreno-Zabaleta R, Arenas-Valls N, Salvador-Maya MA, Algarra-Lucas C, et al. Neurological complications in critical patients with COVID-19. Neurologia 2020;35:621-627

33. Chougar L, Shor N, Weiss N, Galanaud D, Leclercq D, Mathon B, et al. Retrospective observational study of brain MRI findings in patients with acute SARS-CoV-2 infection and neurologic manifestations. Radiology 2020;297:E313-E323

34. Eliezer M, Hamel AL, Houdart E, Herman P, Housset J,
Jourdaine C, et al. Loss of smell in patients with COVID-19: MRI data reveal a transient edema of the olfactory clefts. Neurology 2020;95:e3145-e3152

35. Hernández-Fernández $F$, Sandoval Valencia $H$, BarbellaAponte RA, Collado-Jiménez R, Ayo-Martín 0́, Barrena C, et al. Cerebrovascular disease in patients with COVID-19: neuroimaging, histological and clinical description. Brain 2020;143:3089-3103

36. Kandemirli SG, Dogan L, Sarikaya ZT, Kara S, Akinci C, Kaya D, et al. Brain MRI findings in patients in the intensive care unit with COVID-19 infection. Radiology 2020;297:E232-E235

37. Nawabi J, Morotti A, Wildgruber M, Boulouis G, Kraehling $\mathrm{H}$, Schlunk $\mathrm{F}$, et al. Clinical and imaging characteristics in patients with SARS-CoV-2 infection and acute intracranial hemorrhage. J Clin Med 2020;9:2543

38. Malhotra K, Gornbein J, Saver JL. Ischemic strokes due to large-vessel occlusions contribute disproportionately to stroke-related dependence and death: a review. Front Neurol 2017;8:651

39. Dozois A, Hampton L, Kingston CW, Lambert G, Porcelli TJ, Sorenson D, et al. PLUMBER study (prevalence of large vessel occlusion strokes in Mecklenburg county emergency response). Stroke 2017;48:3397-3399

40. Smith WS, Lev MH, English JD, Camargo EC, Chou M, Johnston $\mathrm{SC}$, et al. Significance of large vessel intracranial occlusion causing acute ischemic stroke and TIA. Stroke 2009;40:38343840

41. Rennert RC, Wali AR, Steinberg JA, Santiago-Dieppa DR, Olson SE, Pannell JS, et al. Epidemiology, natural history, and clinical presentation of large vessel ischemic stroke. Neurosurgery 2019;85:S4-S8

42. Beyrouti R, Adams ME, Benjamin L, Cohen H, Farmer SF, Goh $Y Y$, et al. Characteristics of ischaemic stroke associated with COVID-19. J Neurol Neurosurg Psychiatry 2020;91:889-891

43. Kremer S, Lersy F, de Sèze J, Ferré JC, Maamar A, Carsin-Nicol $B$, et al. Brain MRI findings in severe COVID-19: a retrospective observational study. Radiology 2020;297:E242-E251

44. Bilinska K, Jakubowska P, Von Bartheld CS, Butowt R. Expression of the SARS-CoV-2 entry proteins, ACE2 and TMPRSS2, in cells of the olfactory epithelium: identification of cell types and trends with age. ACS Chem Neurosci 2020;11:1555-1562 